\title{
A Family of High-Performance Matrix Multiplication Algorithms
}

\author{
John A. Gunnels ${ }^{1}$, Greg M. Henry ${ }^{2}$, and Robert A. van de Geijn ${ }^{1}$ \\ 1 Department of Computer Sciences, The University of Texas, Austin, TX 78712, \\ \{gunnels, rvdg\}@cs.utexas.edu, \\ WWW home page: http://www.cs.utexas.edu/users/\{gunnels,rvdg\}/ \\ 2 Intel Corp., Bldg EY2-05, 5350 NE Elam Young Pkwy, Hillsboro, OR 97124-6461, \\ greg.henry@intel.com, \\ WWW home page: http://www.cs.utk.edu/ ghenry/
}

\begin{abstract}
During the last half-decade, a number of research efforts have centered around developing software for generating automatically tuned matrix multiplication kernels. These include the PHiPAC project and the ATLAS project. The software endproducts of both projects employ brute force to search a parameter space for blockings that accommodate multiple levels of memory hierarchy. We take a different approach: using a simple model of hierarchical memories we employ mathematics to determine a locally-optimal strategy for blocking matrices. The theoretical results show that, depending on the shape of the matrices involved, different strategies are locally-optimal. Rather than determining a blocking strategy at library generation time, the theoretical results show that, ideally, one should pursue a heuristic that allows the blocking strategy to be determined dynamically at run-time as a function of the shapes of the operands. When the resulting family of algorithms is combined with a highly optimized inner-kernel for a small matrix multiplication, the approach yields performance that is superior to that of methods that automatically tune such kernels. Preliminary results, for the Intel Pentium (R) III processor, support the theoretical insights.
\end{abstract}

\section{Introduction}

Research in the development of linear algebra libraries has recently shifted to the automatic generation and optimization of the matrix multiplication kernels. The underlying idea is that many linear algebra operations can be implemented in terms of matrix multiplication [2106] and thus it is this operation that should be highly optimized on different platforms. Since the coding effort required to achieve this is considerable, especially when multiple layers of cache are involved, the general consensus is that this process should be automated.

In this paper, we develop a theoretical framework that (1) suggests a formula for the block sizes that should be used at each level of the memory hierarchy, and (2) restricts the possible loop orderings to a specific family of algorithms for matrix multiplication. We show how to use these results to build highly optimized matrix multiplication implementations that utilize the caches in a locally-optimal fashion. The results could be equally well used to limit the search space that must be examined by packages that automatically tune such kernels. 
The current pursuit of highly optimized matrix kernels constructed by coding in a high-level programming language started with the implementation of the FORTRAN implementation of Basic Linear Algebra Subprograms (BLAS) 4] for the IBM POWER2 (TM) [1. Subsequently, the PHiPAC project [3] demonstrated that high-performance matrix multiplication kernels can be written in $\mathrm{C}$ and that code generators could be used to automatically generate many different blockings, allowing automatic tuning. Next, the ATLAS project [11] extended these ideas by reducing the kernel that is called once matrices are massaged to be in the L1 cache into one specific case: $C=A^{T} B+\beta C$ for small matrices $A, B$, and $C$ and by reducing the space searched for optimal blockings. Furthermore it marketed the methodology allowing it to gain wide-spread acceptance and igniting the current trend in the linear algebra community towards automatically tuned libraries. Finally, there has been a considerable recent interest in recursive algorithms and recursive data structures. The idea here is that by recursively partitioning the operands, blocks that fit in the different levels of the caches will automatically be encountered [8]. By storing matrices recursively, blocks that are encountered during the execution of the recursive algorithms will be in contiguous memory [7/9].

Other work closely related to this topic is discussed in other papers presented as part of this session of the conference.

\section{Notation and Terminology}

\subsection{Special Cases of Matrix Multiplication}

The general form of a matrix multiply is $C \leftarrow \alpha A B+\beta C$ where $C$ is $m \times n, A$ is $m \times k$, and $B$ is $k \times n$. We will use the following terminology when referring to a matrix multiply when two dimensions are large and one is small:

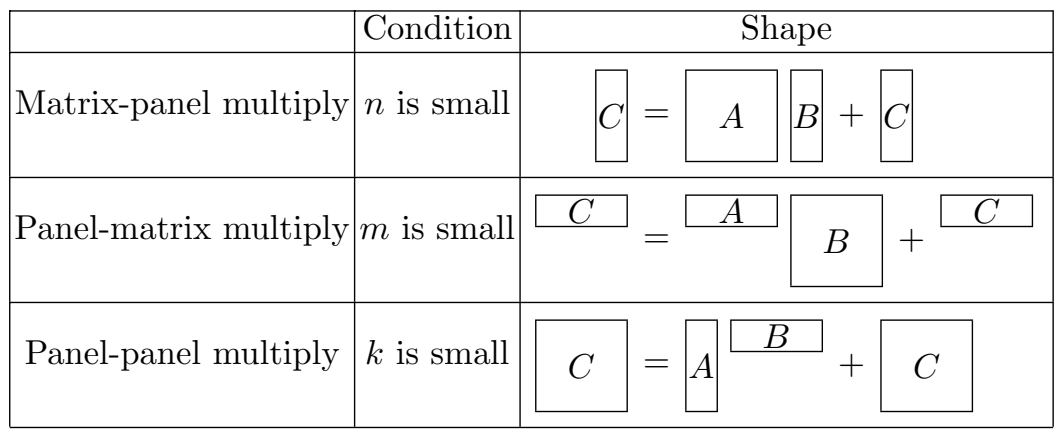

The following observation will become key to understanding concepts encountered in the rest of the paper: Partition $X=\left(X_{1}|\cdots| X_{N_{X}}\right)=\left(\begin{array}{c}\frac{\hat{X}_{1}}{\vdots} \\ \frac{\hat{X}_{M_{X}}}{n_{j}}\end{array}\right)$ for $X \in\{A, B, C\}$, where $C_{j}$ is $m \times n_{j}, \hat{C}_{i}$ is $m_{i} \times n, A_{p}$ is $m \times k_{p}, \hat{A}_{i}$ is $m_{i} \times k$, $B_{j}$ is $k \times n_{j}$, and $\hat{B}_{p}$ is $k_{p} \times n$. Then $C \leftarrow A B+C$ can be achieved by 


\begin{tabular}{|c|c|c|c|c|c|}
\hline $\begin{array}{l}\text { multiple } \\
\text { matrix-panel } \\
\text { multiplies: }\end{array}$ & $\begin{array}{l}C_{j} \leftarrow A B_{j}+C_{j} \\
\text { for } j=1, \ldots, N_{C}\end{array}$ & $C_{1} C_{2} C_{3}$ & $+=$ & $A$ & $B_{1} B_{1} B_{1}$ \\
\hline $\begin{array}{l}\text { multiple } \\
\text { panel-matrix } \\
\text { multiplies: }\end{array}$ & $\begin{array}{l}\hat{C}_{i} \leftarrow \hat{A}_{i} B+\hat{C}_{i} \\
\text { for } i=1, \ldots, M_{C}\end{array}$ & $\begin{array}{l}\hat{C}_{1} \\
\hat{C}_{2} \\
\hat{C}_{3} \\
\end{array}$ & $+=$ & $\begin{array}{l}\hat{A}_{1} \\
\hat{A}_{2} \\
\hat{A}_{3} \\
\end{array}$ & $B$ \\
\hline $\begin{array}{l}\text { multiple } \\
\text { panel-panel } \\
\text { multiplies }\end{array}$ & $C \leftarrow \sum_{p}^{N_{A}} A_{p} \hat{B}_{p}+C$ & $C$ & $+=$ & \begin{tabular}{ll|l}
$A_{1}$ & $A_{2}$ & $A_{3}$
\end{tabular} & $\begin{array}{l}\frac{\hat{B}_{1}}{\hat{B}_{2}} \\
\hat{B}_{3}\end{array}$ \\
\hline
\end{tabular}

\section{$2.2 \quad$ A Cost Model for Hierarchical Memories}

The memory hierarchy of a modern microprocessor is often viewed as a pyramid: At the top of the pyramid, there are the processor registers, with extremely fast access. At the bottom, there are disks and even slower media. As one goes down the pyramid, while the financial cost of memory decreases, the amount of memory increases along with the time required to access that that memory.

We will model the above-mentioned hierarchy naively as follows: (1) The memory hierarchy consists of $H$ levels, indexed $0, \ldots, H-1$. Level 0 corresponds to the registers. We will often denote the $i$ th level by $L_{i}$. Notice that on a typical current architecture $L_{1}$ and $L_{2}$ correspond the level 1 and level 2 data caches and $L_{3}$ corresponds to RAM. (2) Level $h$ of the memory hierarchy can store $S_{h}$ floating-point numbers. Generally $S_{0} \leq S_{1} \leq \cdots \leq S_{H-1}$. (3) Loading a floatingpoint number stored in level $h+1$ to level $h$ costs time $\rho_{h}$. We will assume that $\rho_{0}<\rho_{1}<\cdots<\rho_{H-1}$. (4) Storing a floating-point number from level $h$ to level $h+1$ costs time $\sigma_{h}$. We will assume that $\sigma_{0}<\sigma_{1}<\cdots<\sigma_{H-1}$. (5) If $m_{h} \times n_{h}$ matrix $C, m_{h} \times k_{h}$ matrix $A$, and $k_{h} \times n_{h}$ matrix $B$ are all stored in level $h$ of the memory hierarchy then forming $C \leftarrow A B+C$ costs time $2 m_{h} n_{h} k_{h} \gamma_{h}$. (Notice that $\gamma_{h}$ will depend on $m_{h}, n_{h}$, and $k_{h}$ ).

\section{Building-Blocks for Matrix Multiplication}

Consider the matrix multiplication $C \leftarrow A B+C$ where $m_{h+1} \times n_{h+1}$ matrix $C, m_{h+1} \times k_{h+1}$ matrix $A$, and $k_{h+1} \times n_{h+1}$ matrix $B$ are all stored in $L_{h+1}$. Let us assume that somehow an efficient matrix multiplication kernel exists for matrices stored in $L_{h}$. In this section, we develop three distinct approaches for matrix multiplication kernels for matrices stored in $L_{h+1}$.

Partition

$$
C=\left(\begin{array}{c|c|c}
C_{11} & \cdots & C_{1 N} \\
\hline \vdots & & \vdots \\
\hline C_{M 1} & \cdots & C_{M N}
\end{array}\right), A=\left(\begin{array}{c|c|c}
A_{11} & \cdots & A_{1 K} \\
\hline \vdots & & \vdots \\
\hline A_{M 1} & \cdots & A_{M K}
\end{array}\right), \text { and } B=\left(\begin{array}{c|c|c}
B_{11} & \cdots & B_{1 N} \\
\hline \vdots & & \vdots \\
\hline B_{K 1} & \cdots & B_{K N}
\end{array}\right)
$$




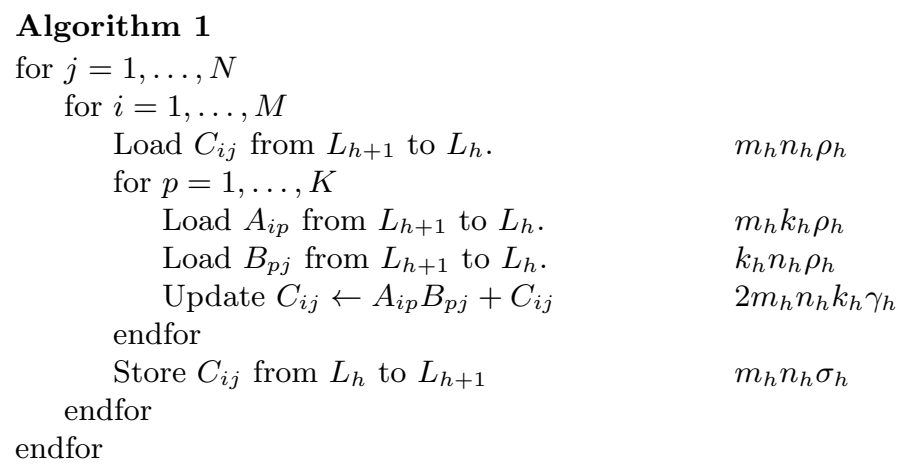

Fig. 1. Multiple panel-panel multiply based blocked matrix multiplication.

where $C_{i j}$ is $m_{h} \times n_{h}, A_{i p}$ is $m_{h} \times k_{h}$, and $B_{p j}$ is $k_{h} \times n_{h}$. The objective of the game will be to determine optimal $m_{h}, n_{h}$, and $k_{h}$.

\subsection{Multiple Panel-Panel Multiplies in $L_{h}$}

Noting that $C_{i j} \leftarrow \sum_{p=1}^{K} A_{i p} B_{p j}+C_{i j}$, let us consider the algorithm in Fig. 1 for computing the matrix multiplication. In that figure the costs of the various operations are shown to the right. The order of the outer-most loops is irrelevant to the analysis.

The cost for updating $C$ is given by

$$
m_{h+1} n_{h+1}\left(\rho_{h}+\sigma_{h}\right)+m_{h+1} n_{h+1} k_{h+1} \frac{\rho_{h}}{n_{h}}+m_{h+1} n_{h+1} k_{h+1} \frac{\rho_{h}}{m_{h}}+2 m_{h+1} n_{h+1} k_{h+1} \gamma_{h}
$$

Since it equals $2 m_{h+1} n_{h+1} k_{h+1}$, solving for $\gamma_{h+1}$, the effective cost per floatingpoint operation at level $L_{h+1}$, yields $\gamma_{h+1}^{P P}=\frac{\rho_{h}+\sigma_{h}}{2 k_{h+1}}+\frac{\rho_{h}}{2 n_{h}}+\frac{\rho_{h}}{2 m_{h}}+\gamma_{h}$. The question now is how to find the $m_{h}, n_{h}$, and $k_{h}$ that minimize $\gamma_{h+1}$ under the constraint that $C_{i j}, A_{i k}$ and $B_{k j}$ all fit in $L_{h}$, i.e., $m_{h} n_{h}+m_{h} k_{h}+n_{h} k_{h} \leq S_{h}$. The smaller $k_{h}$, the more space in $L_{h}$ can be dedicated to $C_{i j}$ and thus the smaller the fractions $\rho_{h} / m_{h}$ and $\rho_{h} / n_{h}$ can be made. A good strategy is thus to let essentially all of $L_{h}$ be dedicated to $C_{i j}$, i.e., $m_{h} n_{h} \approx S_{h}$. The minimum is then attained when $m_{h} \approx n_{h} \approx \sqrt{S_{h}}$.

Notice that it suffices to have $m_{h+1}=m_{h}$ or $n_{h+1}=n_{h}$ for the above cost of $\gamma_{h+1}$ to be minimized. Thus, the above already holds for the special cases depicted in (1) and (2), i.e., when $N=1$ and $M=1$ in (4), respectively.

The innermost loop in Alg. 1 implements multiple panel-panel multiplies since $k_{h}$ is assumed to be small relative to $m_{h}$ and $n_{h}$. Hence the name of this section.

\subsection{Multiple Matrix-Panel Multiplies in $\boldsymbol{L}_{h}$}

Moving the loops over $l$ and $i$ to the outside we obtain the algorithm in Fig. 2 (left). Performing an analysis similar to that given in Section 3.1 the 


\section{Algorithm 2}

for $p=1, \ldots, K$

for $i=1, \ldots, M$

Load $A_{i p}$ from $L_{h+1}$ to $L_{h}$.

for $j=1, \ldots, N$

Load $C_{i j}$ from $L_{h+1}$ to $L_{h}$.

Load $B_{p j}$ from $L_{h+1}$ to $L_{h}$.

Update $C_{i j} \leftarrow A_{i p} B_{p j}+C_{i j}$ endfor

Store $C_{i j}$ from $L_{h}$ to $L_{h+1}$

endfor

endfor

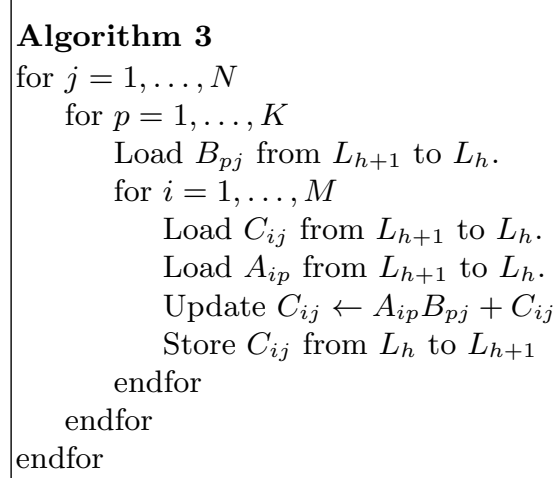

Fig. 2. Multiple matrix-panel (left) and panel-matrix (right) multiply based blocked matrix multiplication.

effective cost of a floating-point operation is now given by $\gamma_{h+1}^{M P}=\frac{\rho_{h}}{2 n_{h+1}}+$ $\frac{\rho_{h}+\sigma_{h}}{2 k_{h}}+\frac{\rho_{h}}{2 m_{h}}+\gamma_{h}$.

Again, the question is how to find the $m_{h}, n_{h}$, and $k_{h}$ that minimize $\gamma_{h+1}$ under the constraint that $C_{i j}, A_{i k}$ and $B_{k j}$ all fit in $L_{h}$, i.e., $m_{h} n_{h}+m_{h} k_{h}+$ $n_{h} k_{h} \leq S_{h}$. Note that the smaller $n_{h}$, the more space in $L_{h}$ can be dedicated to $A_{i l}$ and thus the smaller the fractions $\left(\rho_{h}+\sigma_{h}\right) / 2 k_{h}$ and $\rho_{h} / 2 m_{h}$ can be made. A good strategy is thus to let essentially all of $L_{h}$ be dedicated to $A_{i l}$, i.e., $m_{h} k_{h} \approx S_{h}$. The minimum is then attained when $m_{h} \approx k_{h} \approx \sqrt{S_{h}}$.

Notice that it suffices to have $m_{h+1}=m_{h}$ or $k_{h+1}=k_{h}$ for the above cost of $\gamma_{h+1}$ to be minimized. In other words, the above holds for the special cases depicted in (2) and (3), i.e., when $M=1$ and $K=1$ in (4), respectively.

The innermost loop in Alg. 2 implements multiple matrix-panel multiplies since $n_{h}$ is small relative to $m_{h}$ and $k_{h}$. Thus the name of this section.

\subsection{Multiple Panel-Matrix Multiplies in $L_{h}$}

Finally, moving the loops over $p$ and $j$ to the outside we obtain the algorithm given in Fig. 2 (right). This time, the effective cost of a floating-point operation is given by $\gamma_{h+1}^{P M}=\frac{\rho_{h}}{2 m_{h+1}}+\frac{\rho_{h}+\sigma_{h}}{2 k_{h}}+\frac{\rho_{h}}{2 n_{h}}+\gamma_{h}$.

Again, the question is how to find the $m_{h}, n_{h}$, and $k_{h}$ that minimize $\gamma_{h+1}$ under the constraint that $C_{i j}, A_{i k}$ and $B_{k j}$ all fit in $L_{h}$, i.e., $m_{h} n_{h}+m_{h} k_{h}+$ $n_{h} k_{h} \leq S_{h}$. Note that the smaller $m_{h}$, the more space in $L_{h}$ can be dedicated to $B_{p j}$ and thus the smaller the fractions $\left(\rho_{h}+\sigma_{h}\right) / 2 k_{h}$ and $\rho_{h} / 2 n_{h}$ can be made. A good strategy in this case is to dedicate essentially all of $L_{h}$ to $B_{p j}$, i.e., $n_{h} k_{h} \approx S_{h}$. The minimum is then attained when $n_{h} \approx k_{h} \approx \sqrt{S_{h}}$.

Notice that it suffices to have $n_{h+1}=n_{h}$ and/or $k_{h+1}=k_{h}$ for the above cost of $\gamma_{h+1}$ to be achieved. In other words, the above holds for the special cases depicted in (1) and (3), i.e., when $N=1$ and $K=1$ in (4), respectively. 


\subsection{Summary}

The conclusions to draw from Sections 2.1 and 3.1 3.3 are: (1) There are three shapes of matrix multiplication that one expects to encounter at each level of the memory hierarchy: panel-panel, matrix-panel, and panel-matrix multiplication. (2) If one such shape is encountered at $L_{h+1}$, a locally-optimal approach to utilizing $L_{h}$ will perform multiple instances with one of the other two shapes. (3) Given that multiple instances of a given shape are to be performed, the strategy is to move a submatrix of one of the three operands into $L_{h}$ (we will call this the resident matrix in $L_{h}$ ), filling most of that layer, and to amortize the cost of this data movement by streaming submatrices from the other operands from $L_{h+1}$ to $L_{h}$.

Interestingly enough, the shapes discussed are exactly those that we encountered when studying a class of matrix multiplication algorithms on distributed memory architectures [5]. This is not surprising, since distributed memory is just another layer in the memory hierarchy.

\section{A Family of Algorithms}

We now turn the observations made above into a practical implementation.

High-performance implementations of matrix multiplication typically start with an "inner-kernel". This kernel carefully orchestrates the movement of data in and out of the registers and the computation under the assumption that one or more of the operands are in the L1 cache. For our implementation on the Intel Pentium (R) III processor, the inner-kernel performs the operation $C=A^{T} B+\beta C$ where $64 \times 8$ matrix $A$ is kept in the L1 cache. Matrices $B$ and $C$ have a large number of columns, which we view as multiple-panels, with each panel of width one. Thus, our inner-kernel performs a multiple matrix-panel multiply (MMP) with a transposed resident matrix $A$. The technical reasons why this particular shape was selected go beyond the scope of this paper.

While it may appear that we thus only have one of the three kernels for operation in the L1 cache, notice that for the submatrices with which we compute at that level one can instead compute $C^{T}=B^{T} A+C^{T}$, reversing the role of $A$ and $B$. This simple observation allows us to claim that we also have an innerkernel that performs a multiple panel-matrix multiply (MPM).

Let us introduce a naming convention for a family of algorithms that perform the discussed algorithms at different levels of the memory hierarchy:

$<$ kernel at $L_{3}>-<$ kernel at $L_{2}>-<$ kernel at $L_{1}>$.

For example MPP-MPM-MMP will indicate that the $L_{3}$-kernel uses multiple panel-panel multiplies, calls the $L_{2}$-kernel that uses multiple matrix-panel multiplies, which in turn calls the $L_{1}$-kernel that uses multiple panel-matrix multiplies. Given the constraint that only two of the possible three kernel algorithms are implemented at $L_{1}$, the tree of algorithms in Fig. 3 can be constructed. 


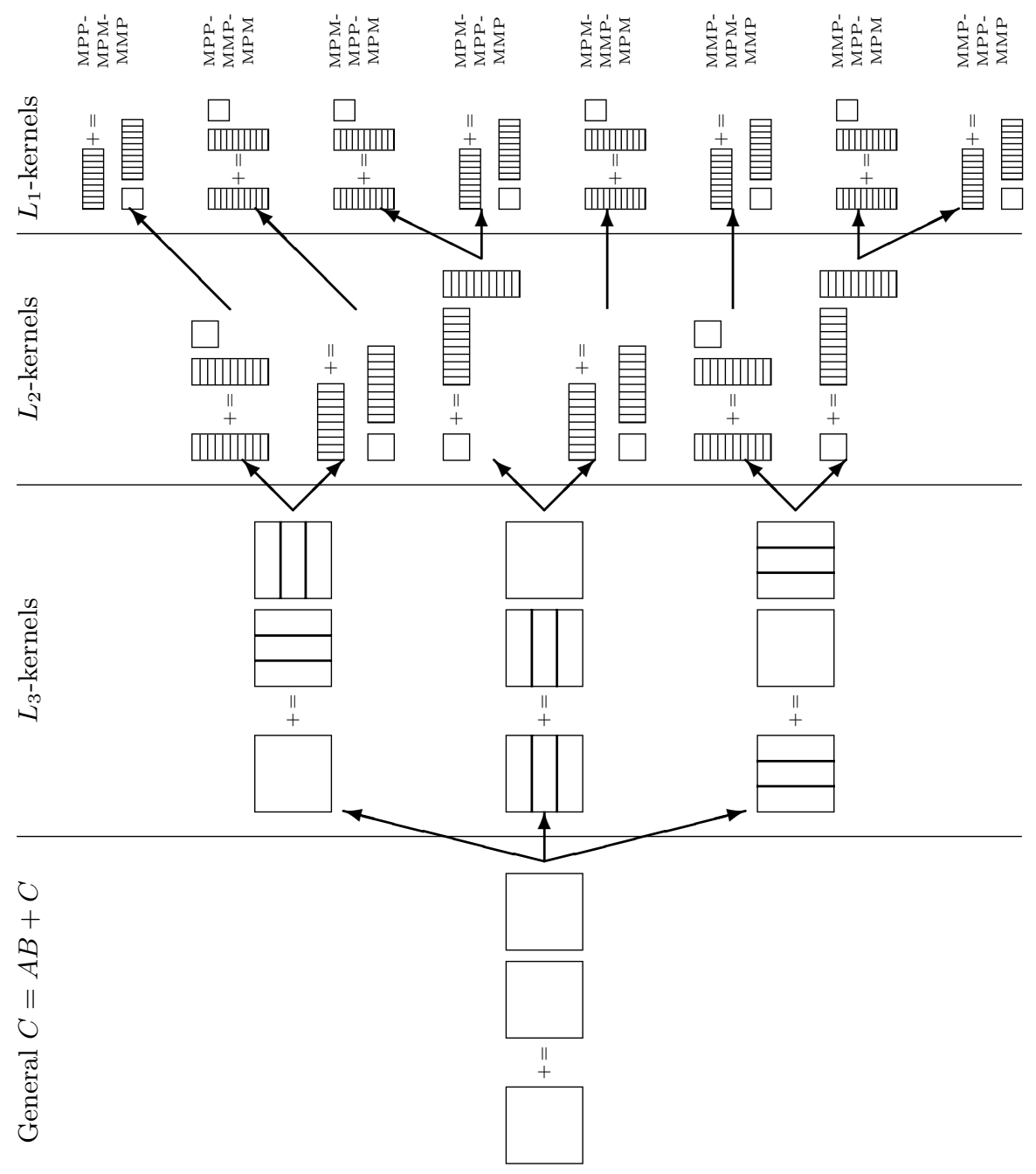

Fig. 3. Possible algorithms for matrices in memory level $L_{3}$ given all $L_{2}$-kernels. 


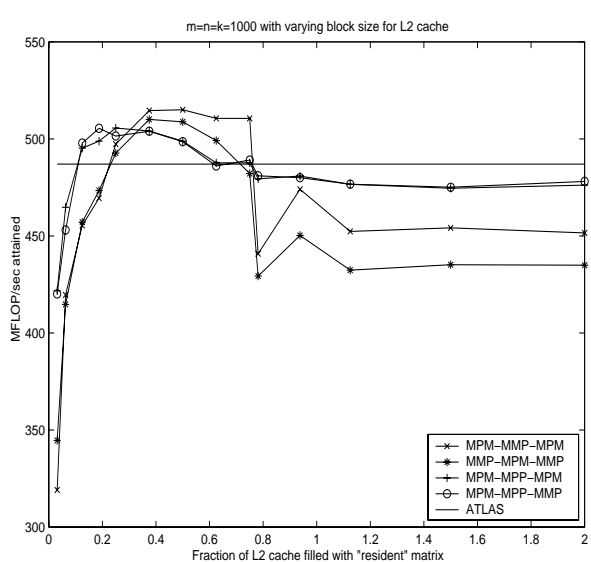

(a)

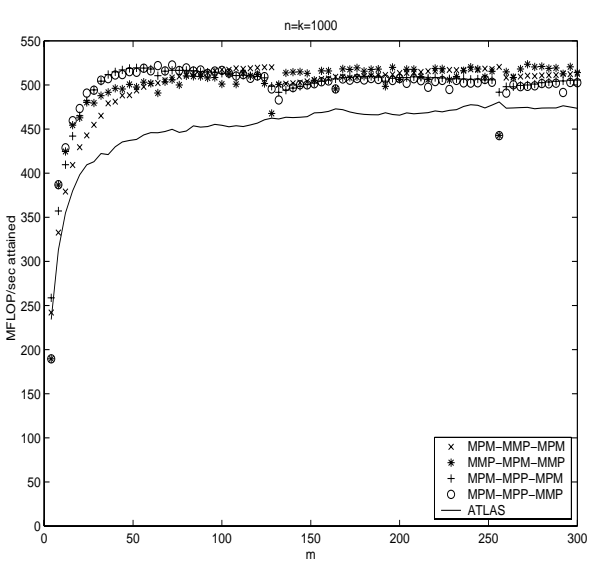

(b)

Fig. 4. Left: Performance for fixed dimensions $m=n=k=1000$ as a function of the size of the resident matrix in the L2 cache. Right: Performance as a function of $m$ when $n=k=1000$.

\section{Performance}

In this section, we report performance attained by the different algorithms. Performance is reported by the rate of computations attained, in millions of floatingpoint operations per second (MFLOP/sec) using 64-bit arithmetic. For the usual matrix dimensions $m, n$, and $k$, we use the operation count $2 m n k$ for the matrix multiplication. We tested performance of the operation $C=C-A B(\alpha=-1$ and $\beta=1$ ) since this is the case most frequently encountered when matrix multiplication is used in libraries such as LAPACK.

We report performance on an Intel Pentium (R) III (650 MHz) processor with a 16 Kbyte L1 data cache and a 256 Kbyte L2 cache running RedHat Linux 6.2. The inner-kernel, which perform the operation $C \leftarrow A^{T} B+\beta C$ with $64 \times 8$ matrix $A$ and $64 \times k$ matrix $B$, was hand-coded using Intel Streaming SIMD Extensions (TM) (SSE). In order to keep the graphs readable, we only report performance for four of the eight possible algorithms. For reference, we report performance of the matrix multiply from ATLAS R3.2, which does not use Intel SSE instructions, for this architecture.

Our first experiment is intended to demonstrate that the block size selected for the matrix that remains resident in the L2 cache has a clear effect on the overall performance of the matrix multiplication routine. In Fig. 4(a) we report performance attained as a function of the fraction of the L2 cache filled with the resident matrix when a matrix multiplication with $k=m=n=1000$ is executed. This experiment tests our theory that reuse of data in the L2 cache impacts overall performance as well as our theory that the resident matrix should occupy "most" of the L2 cache. Note that performance improves as a larger fraction of the L2 cache is filled with the resident matrix. Once the resident matrix fills more than half of the L2 cache, performance starts to deminish. 
This is consistent with the theory which tells us that some of the cache must be used for the matrices that are being streamed from main memory. Once more than $3 / 4$ of the L2 cache is filled with the resident matrix, performance drops significantly. This is consistent with the scenario wherein parts of the other matrices start evicting parts of the resident matrix from the L2 cache. Based on the above experiment, we fix the block size for the resident matrix in the L2 cache to $128 \times 128$, which fills exactly half of this cache, for the remaining experiments.

In Fig. 4(b) we show performance as a function of $m$ when $n$ and $k$ are fixed to be large. There is more information in this graph than we can discuss in this paper. Notice for example that performance of the algorithm that performs multiple panel-matrix multiplies in the $L_{3}$ cache and multiple matrix-panel multiplies in the $L_{2}$ cache, MPM_MMP_MPM, increases as $m$ increases to a multiple of 128. This is consistent with the theory.

For additional and more up-to-date performance graphs, and related discussion, we refer the reader to the ITXGEMM web page mentioned in the conclusion.

\section{Conclusion}

In this paper, theoretical insight was used to motivate a family of algorithms for matrix multiplication on hierarchical memory architectures. The approach attempts to amortize the cost of moving data between memory layers in a fashion that is locally-optimal. Preliminary experimental results on the Intel Pentium (R) III processor appear to support the theoretical results.

Many questions regarding this subject are not addressed in this paper, some due to space limitations. For example, the techniques can be, and have been, trivially extended to the other cases of matrix multiplication: $C \leftarrow \alpha A^{T} B+\beta C$, $C \leftarrow \alpha A B^{T}+\beta C$, and $C \leftarrow \alpha A^{T} B^{T}+\beta C$ by transposing matrices at appropriate stages in the algorithm. Also, while we claim that given different matrix dimensions, $m, n$, and $k$, a different algorithm may be best, we do not address how to choose from the different algorithms. We have developed simple heuristics that yield very satisfactory results. Experiments that support the theory, performed on a number of different architectures, are needed to draw definitive conclusions. The theory should be extended to include a model of cache-replacement policies. How performance is affected by the hand-coded inner-kernel needs to be quantified. We hope to address these issues in a future paper.

Clearly, our techniques can be used to reduce the set of block sizes to be searched at each level of the memory hierarchy. Thus, our techniques could be combined with techniques for automatically generating the inner-kernel and/or an automated search for the optimal block sizes.

More information: http://www.cs.utexas.edu/users/flame/ITXGEMM/.

Acknowledgments: We thank Dr. Fred Gustavson for valuable feedback regarding this project. 


\section{References}

1. R.C. Agarwal, F.G. Gustavson, and M. Zubair. Exploiting functional parallelism of POWER2 to design high-performance numerical algorithms. IBM Journal of Research and Development, 38(5), Sept. 1994.

2. E. Anderson, Z. Bai, C. Bischof, J. Demmel, J. Dongarra, J. Du Croz, A. Greenbaum, S. Hammarling, A. McKenney, S. Ostrouchov, and D. Sorensen. LAPACK Users' Guide - Release 2.0. SIAM, 1994.

3. J. Bilmes, K. Asanovic, C.W. Chin, and J. Demmel. Optimizing matrix multiply using PHiPAC: a portable, high-performance, ANSI C coding methodology. In Proceedings of the International Conference on Supercomputing. ACM SIGARC, July 1997.

4. Jack J. Dongarra, Jeremy Du Croz, Sven Hammarling, and Iain Duff. A set of level 3 basic linear algebra subprograms. ACM Trans. Math. Soft., 16(1):1-17, March 1990.

5. John Gunnels, Calvin Lin, Greg Morrow, and Robert van de Geijn. A flexible class of parallel matrix multiplication algorithms. In Proceedings of First Merged International Parallel Processing Symposium and Symposium on Parallel and Distributed Processing (1998 IPPS/SPDP '98), pages 110-116, 1998.

6. John A. Gunnels and Robert A. van de Geijn. Formal methods for highperformance linear algebra libraries. In Ronald F. Boisvert and Ping Tak Peter Tang, editors, The Architecture of Scientific Software. Kluwer Academic Press, 2001.

7. F. Gustavson, A. Henriksson, I. Jonsson, B. Kågström, and P. Ling. Recursive blocked data formats and BLAS's for dense linear algebra algorithms. In B. Kågström et al., editor, Applied Parallel Computing, Large Scale Scientific and Industrial Problems, volume 1541 of Lecture Notes in Computer Science, pages 195-206. Springer-Verlag, 1998.

8. F. G. Gustavson. Recursion leads to automatic variable blocking for dense linearalgebra algorithms. IBM Journal of Research and Development, 41(6):737-755, November 1997.

9. Greg Henry. BLAS based on block data structures. Theory Center Technical Report CTC92TR89, Cornell University, Feb. 1992.

10. B. Kågström, P. Ling, and C. Van Loan. GEMM-based level 3 BLAS: High performance model implementations and performance evaluation benchmark. Technical Report CS-95-315, Univ. of Tennessee, Nov. 1995.

11. R. Clint Whaley and Jack J. Dongarra. Automatically tuned linear algebra software. In Proceedings of SC98, Nov. 1998. 